Letter to the Editor

DOI: $10.2478 / 10004-1254-65-2014-2570$

\title{
A survey of professional satisfaction among anaesthesiologists in Serbia
}

\author{
Ljiljana Gvozdenović, Tatjana Batak, Dejan Ivanov, and Radovan Cvijanović \\ Medical University Novi Sad, Clinical Center Vojvodina, Novi Sad, Serbia
}

We would like to briefly address our recent findings regarding professional satisfaction among anaesthesiologists in Serbia. It is generally wellknown among physicians that the life expectancy of anaesthesiologists is smaller than in other specialties (1). Many resort to the use of cigarettes, drugs, alcohol (2). The number of suicides among anaesthesiologists is significantly larger in comparison with other physicians $(3,4)$. Needless to say, the level of professional satisfaction is in a direct link with the quality of patient care they are able to provide.

To investigate whether there is a lack of satisfaction among anaesthesiologists in our country, we conducted a study aimed at determining if anaesthesiologists in Serbian tertiary hospitals are under chronic stress after 24-hour on-call shifts; if they endure high levels of anxiety; and whether there is a connection between their personality type and alcohol and drug abuse. The study included 60 anaesthesiologists who worked in specialised surgical tertiary health institutions 24 hours on-call. The survey was voluntary and anonymous. All of the physicians involved in the study completed a burnout questionnaire (Oldenburg Burnout Inventory). The mean age of the physicians was 43.1 years $(\mathrm{SD}=8.1$, range $29-63)$. There was no statistically significant difference in the average age of the physicians in relation to the analysed groups (on call 44.1 years: not on call 42.2 years). Of the physicians on call, $33.3 \%$ were over 40 years of age. Both groups comprised more women than men (on call: $67 \%$ women; not on call: $77 \%$ women) and the distribution by sex was not different in the groups of physicians not on call. As much as $90 \%$ of the anaesthesiologists were on call four or more times per month. They smoked and drank alcohol more than the other doctors in the control group. Altogether $77 \%$ of anaesthesiologists could not rest appropriately after an on-call shift, and if their sleep was interrupted, they needed considerably more time to fall asleep again. Anxiety decreased after taking days off. Stable extraverts had an emotionally stable personality type with the lowest anxiety level. A total of $76.5 \%$ of male and $55.8 \%$ female physicians consumed cigarettes, alcohol, and/or drugs. This difference was not statistically significant. A total of $70.6 \%$ of male and $34.9 \%$ of female anaesthesiologist used cigarettes and/or alcohol. This difference was statistically significant $(p=0.027)$. Several studies have considered the necessary strategies in detecting signs of burnout and concluded that better working conditions reduced stress on the employees; however, the support of colleagues at work and the heads of the hospitals was as equally important as family support systems (5-8).

There are stress conditions and burnout amongst Serbian anaesthesiologists. The prevalence of depersonalisation was extremely high in the studied sample. In addition to effects on the health of the anaesthesiologists, burnout and depression may also affect patient care and safety.

\section{REFERENCES}

1. Katz JD. Do anesthesiologists die at a younger age than other physicians? Age-adjusted death rates. Anesth Analg 2004;98:1111-3. doi: 10.1213/01.ANE.0000105879.02463.1F

2. Bryson EO, Silverstein JH. Addiction and substance abuse in anesthesiology. Anesthesiology 2008;109:905-17. doi: 10.1097/ALN.0b013e3181895bc1

3. Nyssen AS, Hansez I, Baele P, Lamy M, De Keyser V. Occupational stress and burnout in anaesthesia. Br J Anaesth 2003;90:333-7. doi: 10.1093/bja/aeg058

4. Gaszynska E, Stankiewicz-Rudnicki M, Szatko F, Wieczorek A, Gaszynski T. Life satisfaction and work-related 
satisfaction among anesthesiologists in Poland. Scientific World J 2014;2014:601865. doi: 10.1155/2014/601865

5. Firth-Cozens J, Payne RL, editors. Stress in Health Professionals: Psychological and Organisational Causes and Interventions. Chichester: John Wiley and Sons; 1999.

6. Rama-Maceiras P, Parente S, Crank P. Job satisfaction, stress and burnout in anaesthesia: relevant topics for anaesthesiologists and healthcare managers? Eur J Anaesthesiol 2012;29:311-9. doi: 10.1097/ EJA.0b013e328352816d
7. Abut YC, Kitapcioglu D, Erkalp K, Toprak N, Boztepe A, Sivrikaya U, Palsy I, Gur EK, Eren G, Bilen A. Job burnout in 159 anesthesiology trainees. Saudi J Anaesth 2012;6:4651. doi: 10.4103/1658-354X.93059

8. de Oliveira GS Jr, Chang R, Fitzgerald PC, Almeida MD, Castro-Alves LS, Ahmad S, McCarthy RJ. The prevalence of burnout and depression and their association with adherence to safety and practice standards: a survey of United States anesthesiology trainees. Anesth Analg 2013;117:18293. doi: 10.1213/ANE.0b013e3182917da9

\section{CORRESPONDING AUTHOR:}

Ljiljana Gvozdenović

Clinical Center of Vojvodina

Novi Sad, Serbia

E-mail:profgvozdenovic2010@hotmail.com 